\title{
Seabed propagation of ULF/ELF electromagnetic fields from harmonic dipole sources located on the seafloor
}

\author{
A. C. Fraser-Smith, ${ }^{1}$ A. S. Inan, ${ }^{2}$ O. G. Villard, Jr., ${ }^{1}$ and R. G. Joiner ${ }^{3}$
}

(Received February 29, 1988; accepted May 11, 1988.)

\begin{abstract}
The amplitudes of the quasi-static electromagnetic fields generated at points on the seafloor by harmonic dipole sources (vertically directed magnetic dipoles, horizontally directed magnetic dipoles, vertically directed electric dipoles, and horizontally directed electric dipoles) also located on the seafloor are computed using a numerical integration technique. The primary purpose of these computations is to obtain field amplitudes that can be used in undersea communication studies. An important secondary purpose is to examine the enhancements of the fields produced at moderate to large distances by the presence of the relatively less conducting seafloor, as compared with the fields produced at the same distances in a sea of infinite extent, for frequencies in the ULF/ELF bands (frequencies less than $3 \mathrm{kHz}$ ). These latter enhancements can be surprisingly large, with increases of 4 orders of magnitude or more being typical at distances of $\mathbf{2 0}$ seawater skin depths.
\end{abstract}

\section{INTRODUCTION}

Because of the high attenuation involved $(55 \mathrm{~dB} /$ wavelength), communication through seawater by means of freely propagating electromagnetic waves is difficult to accomplish and is usually restricted to frequencies in the lowest part of the radio band, where the wavelengths are largest, and to ranges that are short in comparison to those that can be achieved at the same frequencies above the sea surface. While it does not yet appear possible to avoid this high attenuation when the transmitter and receiver are both deeply immersed and separated from interfaces with other media by many seawater skin depths, a number of studies have suggested that increased range can be achieved by locating the submerged transmitter and receiver near to the sea surface and utilizing the "up-over-and-down," or "surface," mode of propagation [e.g., Moore and Blair, 1961; Hansen, 1963; Moore, 1967; Bubenik and Fraser-Smith, 1978]. In this mode the major part of the propagation path is through air, a low-loss medium, and increased range results. Less well known is the "down-under-and-up," or "seabed,"

\footnotetext{
'Space, Telecommunications, and Radioscience Laboratory, Stanford University, Stanford, California.

${ }^{2}$ Department of Electrical and Electronics Engineering, Bilkent University, Ankara.

${ }^{3}$ Office of Naval Research, Arlington, Virginia.

Copyright 1988 by the American Geophysical Union.

Paper number 8S0356.

0048-6604/88/008S-0356\$08.00
}

mode of propagation [Mott and Biggs, 1963; Coggon and Morrison, 1970; Frieman and Kroll, 1973; Bostick et al., 1978; Bubenik and Fraser-Smith, 1978; Inan, 1984; King et al., 1986; King, 1986; Inan et al., 1986]. The seabed, being electrically conducting, has nominally the same $55 \mathrm{~dB} /$ wavelength rate of attenuation for propagating electromagnetic fields as does seawater, but because its electrical conductivity is less, and possibly much less, than that of seawater, the wavelength is larger and the attenuation per unit distance is smaller. Some of the properties of this mode were studied by Bubenik and Fraser-Smith [1978] for a transmitter and receiver located at points equidistant between the surface and floor of a sea one seawater skin depth deep. We now extend this earlier work by considering specifically the increased propagation range that might be achieved by placing the transmitter and receiver directly on the seafloor and making full use of the seabed mode. As we will show, substantial increases in range can result.

This work is essentially a continuation of a recent study, reported by Inan et al. [1986], on the enhancements and other changes produced in the ULF/ELF fields generated along the seafloor by long currentcarrying cables also located on the seafloor. Also relevant is the article by Fraser-Smith et al. [1987] describing large amplitude changes in dipole fields induced by the seabed under different circumstances from those investigated here.

Until the last few years it was difficult to evaluate many of the expressions for the field components produced along a seafloor by harmonic dipole 
sources located on the seafloor without either making major simplifying assumptions, and thus obtaining approximate (sometimes very approximate) values for the field components, or being forced to use numerical integration techniques, which can be difficult to implement but which can give accurate field values over wide ranges of frequency and distance. Our approach to computing dipole fields has been to use a numerical integration technique, and that is the method we have used in this work to obtain the field values. However, a substantial analytical advance has recently been made by $R$. W. P. King and his coworkers in evaluating the fields produced along a seafloor, with the result that a number of new analytical expressions of varying degrees of approximation are now available for the field components [e.g., King and Brown, 1984; King, 1985a, b]. We find that there is good agreement between the field values we compute and the field values computed from King's least approximate expressions within their ranges of applicability. We also find good agreement, in fact agreement to many significant figures, between field values we compute using our numerical integration technique and field values calculated from the exact analytical expressions for certain field components produced by vertical magnetic and horizontal electric dipole sources [Wait, 1952, 1961].

The seabed in our work is represented by a single semi-infinite conducting layer, and thus no attempt is made to take account of the lithospheric duct mode of propagation, concerning which there now exists a considerable literature [e.g., Wait, 1954; Wheeler, 1961; Burrows, 1963; Gabillard et al., 1971; Wait and Spies, 1972a, b, c; Heacock, 1971; Bostick et al., 1978]. If such a duct does exist, as suggested by the literature (but unfortunately not yet adequately tested by experiment), there should be increases in the amplitudes of the electromagnetic fields produced at the receiver above those predicted by our computations. However, because our transmitter and receiver are located on the seafloor, and therefore some distance above the probable center of the duct, the increase in the field levels is much more likely to be due to a lower effective seabed conductivity than to any ducting of fields.

Although we refer to the seabed "mode of propagation" in this work, we should point out that the mode is not a clearly defined theoretical entity as are, for example, the transverse magnetic, transverse electric, and transverse electromagnetic modes in wave- guides. We use the term to distinguish the fields propagating primarily through the seabed from those propagating (1) directly through the sea ("direct mode"), (2) in the up-over-and-down mode ("surface mode"), or (3) in a variety of higher-order modes. It is possible to separate the contributions of the various modes to the net fields measured at the receiver, as is done by Bubenik and Fraser-Smith [1978] and King [1985a]. However, by choosing a very deep sea and a transmitter and receiver located on the seafloor, as is done here, we eliminate the up-over-and-down and higher-order modes and minimize the contribution from the direct mode.

This work has application in studies of the properties of the seabed and its electrical conductivity in particular (see Bannister [1968] and Coggon and Morrison [1970] for earlier work on this topic). It also qualifies as a study of seabed effects in general [e.g., Weaver, 1967; Ramaswamy et al., 1972]. However, we believe its primary application is in undersea communication. This application appears promising for the following reasons. First, as we will show in this paper, the seabed propagation mode offers ranges that may be large in comparison with those that can be achieved directly through seawater. Second, the ambient noise level on the seafloor is likely to be much lower than at the sea surface. Third, unlike other possible undersea transmitter-receiver configurations, once a transmitter and receiver have been installed on the seafloor, their positions are unlikely to change in the long term, and they can be comparatively easily located again for maintenance, if necessary. Finally, it would be possible to colocate a chain of receiver-transmitter pairs (analogous to the repeaters used in other communication links) on the seafloor between the primary transmitter and receiver and thus achieve increased range.

\section{CALCULATION OF FIELD COMPONENTS}

Figure 1 shows the geometry employed in the calculation of the electromagnetic field components produced at points on the seafloor by harmonic dipole sources also located on the seafloor. A cylindrical coordinate system $(r, \phi, z)$ is used, and the dipoles, of moment $\boldsymbol{m}$ (magnetic dipoles) or $p$ (electric dipoles) and angular frequency $\omega(\omega=2 \pi f)$, are placed at the origin with the vertical dipole moments directed upward along the $z$ axis and with the horizontal dipole moments directed along the $x$ axis $(\phi=0)$. The seafloor is the plane $z=0$, the region $z>0$ is seawater (permittivity $\varepsilon_{s}$, permeability $\mu_{0}$, conduc- 
tivity $\sigma_{s}$ ), and the region $z \leq 0$ is the seabed, which is assumed to be a homogeneous conducting half-space with permittivity $\varepsilon_{f}$, permeability $\mu_{0}$, and conductivity $\sigma_{f}$. The field components are computed at points $P(r, \phi, 0)$. We consider all major categories of dipole sources: vertically directed electric and magnetic dipoles (VEDs and VMDs, respectively) and horizontally directed electric and magnetic dipoles (HEDs and HMDs, respectively). The field expressions for the four dipole types are as follows:

1. Vertical electric dipole

$$
\begin{aligned}
& E_{r}=\frac{p}{4 \pi \sigma_{s}} \int_{0}^{\infty} F_{2 M} \lambda^{2} d \lambda \\
& E_{z}=\frac{p}{4 \pi \sigma_{s}} \int_{0}^{\infty} \frac{1}{u_{s}} F_{1 M} \lambda^{3} d \lambda \\
& B_{\phi}=\frac{\mu_{0} p}{4 \pi} \int_{0}^{\infty} \frac{1}{u_{s}} F_{2 M} \lambda^{2} d \lambda
\end{aligned}
$$

2. Vertical magnetic dipole

$$
\begin{aligned}
& E_{\phi}=-\frac{i \omega \mu_{0} m}{4 \pi} \int_{0}^{\infty} \frac{1}{u_{s}} F_{2 E} \lambda^{2} d \lambda \\
& B_{r}=\frac{\mu_{0} m}{4 \pi} \int_{0}^{\infty} F_{2 E} \lambda^{2} d \lambda \\
& B_{z}=\frac{\mu_{0} m}{4 \pi} \int_{0}^{\infty} \frac{1}{u_{s}} F_{1 E} \lambda^{3} d \lambda
\end{aligned}
$$

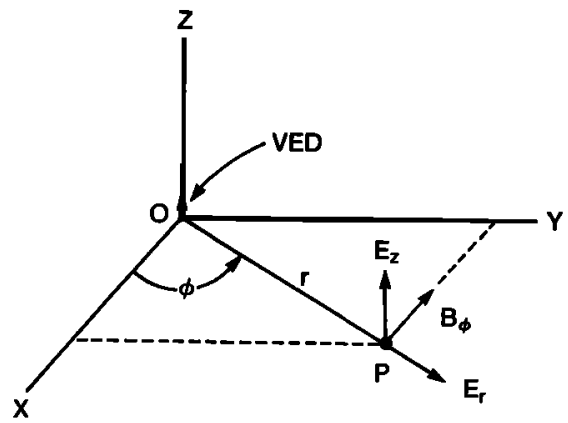

Fig. 1. Coordinate system and geometry used in the computation of the fields produced on the seafloor by harmonic dipole sources. The half space $z<0$ represents the seabed, the surface $z=0$ is the seafloor, and $z>0$ is seawater. The dipoles, illustrated here by the VED, are located at the origin, and the field point $P$ has the coordinates $(r, \phi, 0)$. Both the dipoles and the field point are located in the seawater, but at an infinitesimal distance above the seafloor.
3. Horizontal electric dipole

$$
\begin{aligned}
E_{r} & =\frac{p \cos \phi}{4 \pi \sigma_{s}}\left\{-\int_{0}^{\infty} u_{s} F_{3 M} \lambda d \lambda\right. \\
& \left.+\frac{1}{r}\left[\int_{0}^{\infty} u_{s} F_{4 M} d \lambda-\gamma_{s}^{2} \int_{0}^{\infty} \frac{1}{u_{s}} F_{2 E} d \lambda\right]\right\} \\
E_{\phi} & =\frac{p \sin \phi}{4 \pi \sigma_{s}}\left\{\gamma_{s}^{2} \int_{0}^{\infty} \frac{1}{u_{s}} F_{1 E} \lambda d \lambda\right. \\
& \left.+\frac{1}{r}\left[\int_{0}^{\infty} u_{s} F_{4 M} d \lambda-\gamma_{s}^{2} \int_{0}^{\infty} \frac{1}{u_{s}} F_{2 E} d \lambda\right]\right\} \\
E_{z} & =\frac{p \cos \phi}{4 \pi \sigma_{s}} \int_{0}^{\infty} F_{4 M} \lambda^{2} d \lambda \\
B_{r} & =\frac{\mu_{0} p \sin \phi}{4 \pi}\left\{-\int_{0}^{\infty} F_{1 E} \lambda d \lambda\right. \\
& \left.+\frac{1}{r}\left[\int_{0}^{\infty} F_{2 E} d \lambda-\int_{0}^{\infty} F_{4 M} d \lambda\right]\right\}
\end{aligned}
$$

$$
\begin{gathered}
B_{\phi}=-\frac{\mu_{0} p \cos \phi}{4 \pi}\left\{\int_{0}^{\infty} F_{3 M} \lambda d \lambda\right. \\
\left.+\frac{1}{r}\left[\int_{0}^{\infty} F_{2 E} d \lambda-\int_{0}^{\infty} F_{4 M} d \lambda\right]\right\}
\end{gathered}
$$

$B_{z}=\frac{\mu_{0} p \sin \phi}{4 \pi} \int_{0}^{\infty} \frac{1}{u_{s}} F_{2 E} \lambda^{2} d \lambda$

4. Horizontal magnetic dipole

$$
\begin{aligned}
E_{r} & =\frac{i \omega \mu_{0} m \sin \phi}{4 \pi}\left\{\int_{0}^{\infty} F_{1 M} \lambda d \lambda\right. \\
& \left.+\frac{1}{r}\left[-\int_{0}^{\infty} F_{2 M} d \lambda+\int_{0}^{\infty} F_{4 E} d \lambda\right]\right\} \\
E_{\phi} & =-\frac{i \omega \mu_{0} m \cos \phi}{4 \pi}\left\{-\int_{0}^{\infty} F_{3 E} \lambda d \lambda\right. \\
& \left.+\frac{1}{r}\left[-\int_{0}^{\infty} F_{2 M} d \lambda+\int_{0}^{\infty} F_{4 E} d \lambda\right]\right\}
\end{aligned}
$$

$$
\begin{aligned}
E_{z} & =-\frac{i \omega \mu_{0} m \sin \phi}{4 \pi} \int_{0}^{\infty} \frac{1}{u_{s}} F_{2 M} \lambda^{2} d \lambda \\
B_{r} & =\frac{\mu_{0} m \cos \phi}{4 \pi}\left\{-\int_{0}^{\infty} u_{s} F_{3 E} \lambda d \lambda\right. \\
& \left.+\frac{1}{r}\left[\int_{0}^{\infty} u_{s} F_{4 E} d \lambda-\gamma_{s}^{2} \int_{0}^{\infty} \frac{1}{u_{s}} F_{2 M} d \lambda\right]\right\}
\end{aligned}
$$




$$
\begin{aligned}
B_{\phi} & =\frac{\mu_{0} m \sin \phi}{4 \pi}\left\{\gamma_{s}^{2} \int_{0}^{\infty} \frac{1}{u_{s}} F_{1 M} \lambda d \lambda\right. \\
& \left.+\frac{1}{r}\left[\int_{0}^{\infty} u_{s} F_{4 E} d \lambda-\gamma_{s}^{2} \int_{0}^{\infty} \frac{1}{u_{s}} F_{2 M} d \lambda\right]\right\} \\
B_{z} & =\frac{\mu_{0} m \cos \phi}{4 \pi} \int_{0}^{\infty} F_{4 E} \lambda^{2} d \lambda
\end{aligned}
$$

All the above field quantities are sinusoidal functions of time, and thus, in the representation used here, they are complex quantities with the implicit multiplier $e^{i \omega t}$, and the actual fields are given by the real parts of the expressions. The following equations define the subsidiary variables appearing in the expressions:

$$
\begin{array}{ll}
F_{1 E}=F_{T E}^{(0)} J_{0}(\lambda r) & F_{2 E}=F_{T E}^{(a)} J_{1}(\lambda r) \\
F_{3 E}=F_{T E}^{(b)} J_{0}(\lambda r) & F_{4 E}=F_{T E}^{(b)} J_{1}(\lambda r) \\
F_{1 M}=F_{T M}^{(a)} J_{0}(\lambda r) & F_{2 M}=F_{T M}^{(a)} J_{1}(\lambda r) \\
F_{3 M}=F_{T M}^{(b)} J_{0}(\lambda r) & F_{4 M}=F_{T M}^{(b)} J_{1}(\lambda r)
\end{array}
$$

where

$$
\begin{aligned}
F_{T E}^{(a)}=\frac{2 u_{s}}{u_{s}+u_{f}} & F_{T E}^{(b)}=\frac{2 u_{f}}{u_{s}+u_{f}} \\
F_{T M}^{(a)}=\frac{2 \gamma_{f}^{2} u_{s}}{\gamma_{s}^{2} u_{f}+\gamma_{f}^{2} u_{s}} & F_{T M}^{(b)}=\frac{2 \gamma_{s}^{2} u_{f}}{\gamma_{s}^{2} u_{f}+\gamma_{f}^{2} u_{s}} \\
u_{s}^{2}=\lambda^{2}+\gamma_{s}^{2} & u_{f}^{2}=\lambda^{2}+\gamma_{f}^{2} \\
\gamma_{s}^{2}=i \omega \mu_{0} \sigma_{s} & \gamma_{f}^{2}=i \omega \mu_{0} \sigma_{f}
\end{aligned}
$$

Here $\mu_{0}$ is the permeability of free space $\left(\mu_{0}=4 \pi\right.$ $\times 10^{-7} \mathrm{H} / \mathrm{m}$; the seabed is assumed to be nonmagnetic), and the quantities $\gamma_{s}$ and $\gamma_{f}$ are the propagation constants for the sea and seabed. There is an approximation involved in the two expressions that are given for $\gamma_{s}$ and $\gamma_{f}$. For a general conducting medium $(\mu, \varepsilon, \sigma)$, the full expression for the propagation constant is

$$
\gamma^{2}=-\omega^{2} \mu \varepsilon+i \omega \mu \sigma
$$

but for the conducting media and frequencies of interest in this work (ULF/ELF; frequencies less than $3 \mathrm{kHz}$ ) the displacement current term $\omega^{2} \mu \varepsilon$ may be neglected. This is a common approximation in the computation of electromagnetic fields produced by harmonic dipole sources in the presence of conducting media. It is often made as part of the "quasistatic approximation," which is applicable when the source-receiver distances are much less than a free space wavelength [Kraichman, 1976]. This condition is always well satisfied for the source receiver distances considered in this paper, and thus our data could be said to apply under the conditions of the quasi-static approximation. However, the terminology appears to have little significance when the propagation paths are confined to conducting media.

We evaluated the dipole field expressions numerically, using the techniques described by Bubenik [1977]. The dipole moments were set equal to unity ( $m=1 \mathrm{~A} \mathrm{\textrm {m } ^ { 2 }}$ and $p=1 \mathrm{~A} \mathrm{~m}$ ), and for dipoles of arbitrary moment our field values should be multiplied by the moment to obtain the corresponding field magnitudes. The computations were also carried out in normalized form, to preserve generality and to reduce the computational effort [Bubenik and FraserSmith, 1978; Fraser-Smith and Bubenik, 1979, 1980]. The two important features of this normalization are (1) the seabed conductivity is referred to that of seawater, and (2) distances are measured in units of the seawater skin depth $\delta_{s}$, where

$$
\delta_{s}=\left(2 / \omega \mu_{0} \sigma_{s}\right)^{1 / 2}
$$

As a result of this normalization procedure, frequency and conductivity are removed as explicit variables during evaluation of the field expressions. We use the picotesla as our unit for the magnetic field ( $1 \mathrm{pT}=1 \mathrm{~m} \gamma=10^{-12} \mathrm{~T}$ ), and the electric field data are presented in units of microvolts per meter.

\section{NUMERICAL RESULTS}

Our results consist of amplitude data for (1) the radial and vertical electric field components $E_{r}$ and $E_{z}$, the total electric field $E_{\mathrm{TOT}}$, and the total magnetic field $B_{\text {TOT }}\left(B_{\text {TOT }}=B_{\phi}\right)$ produced by the VED, and (2) the radial and vertical magnetic field components $B_{r}$ and $B_{z}$, the total electric field $E_{\text {TOT }}\left(E_{\text {TOT }}=\right.$ $\left.E_{\phi}\right)$, and the total magnetic field component $B_{\text {TOT }}$ produced by the VMD. We also present (3) amplitude data for the three electric field components $\left(E_{r}\right.$, $\left.E_{\phi}, E_{z}\right)$, three magnetic field components $\left(B_{r}, B_{\phi}, B_{z}\right)$, total electric field $E_{\text {TOT }}$, and total magnetic field $B_{\text {TOT }}$ produced by the HED and HMD at the two principal azimuthal angles $\phi=0^{\circ}$ and $90^{\circ}$. This choice of azimuthal angles simplifies the presentation of the field data; furthermore, as we will now show, it does not significantly limit the applicability of the field data at general azimuthal angles.

Because of the $\sin \phi$ and $\cos \phi$ terms appearing in the equations for the field components produced by 
the two horizontal dipoles (equations (7)-(18)), only one of the horizontal electric components $\left(E_{r}\right.$ and $\left.E_{\phi}\right)$ and one of the horizontal magnetic components $\left(B_{r}\right.$ and $B_{\phi}$ ) are produced by each of the dipoles when $\phi=0^{\circ}$ or $90^{\circ}$ (these nonzero horizontal components are denoted either by $E_{\mathrm{HOR}}$ and $\boldsymbol{B}_{\mathrm{HOR}}$, when there is a vertical electric or magnetic field component present as well, or by $E_{\text {тот }}$ or $B_{\text {TOT }}$, when they are the only electric or magnetic field component; whether they are radial or azimuthal can be determined quickly by noting the azimuthal angle and referring to (7)-(18)). Similarly, only one of the two vertical components $E_{z}$ and $B_{z}$ is produced at each of the two azimuthal angles. As a result, only three basic electric and magnetic field components are produced by the horizontal dipoles when $\phi=0^{\circ}$ and $90^{\circ}$. These two choices of $\phi$ therefore simplify the presentation of numerical field data, but not at the expense of generality, since field amplitudes at an arbitrary $\phi$ can be obtained by multiplying the amplitudes given for $\phi=0^{\circ}$ or $\phi=90^{\circ}$ by the appropriate value of $\cos \phi$ or $\sin \phi$. The choice of which angular function to use is determined by the presence of the function in the applicable equation of the set (7)-(18). For example, suppose amplitudes are required for the three electric field components $E_{r}, E_{\phi}$, and $E_{z}$ produced by the HED at azimuthal angle $\phi$. The amplitudes of $E_{r}$ and $E_{z}$ are found by referring to the HED, $\phi=0^{\circ}$, results and multiplying the appropriate values of $E_{\text {HOR }}$ and $E_{z}$ by $\cos \phi$, which appears in (7) and (9) for the two field components, and the amplitude of $E_{\phi}$ is found by referring to the HED, $\phi=90^{\circ}$, results and multiplying the appropriate $E_{\text {TOт }}$ value by $\sin \phi$, which appears in (8).

The presentation of the field data is similar, but not identical, to presentations used previously by $B u$ benik and Fraser-Smith [1978] and Fraser-Smith and Bubenik [1979, 1980]. First, we present a series of curves that give, in this case, the actual amplitudes of the fields produced on the seafloor by a particular unit moment dipole that is also located on the seafloor. Next, we present additional curves that give the ratios of the amplitudes of the fields produced by the dipole in the presence of the seabed to the amplitudes produced under otherwise identical conditions by the dipole submerged in the sea of infinite depth. These latter curves enable us to identify the changes produced in the fields specifically by the presence of the seabed, since the absence of a seabed effect is indicated by a ratio of unity.

To further illustrate the effects produced on the field quantities by the seabed, the normalized seabed conductivity $\sigma_{f} / \sigma_{s}$ is varied widely, with values in the range 1 (no seabed effect), $0.3,0.1,0.03,0.01,0.003$, 0.001 . It is possible that some materials in a real seabed have a normalized conductivity less than 0.001 , but it is unlikely that the effective overall conductivity will be less than 0.001 , because of the inclusion of the relatively high conductivity sediments close to the floor. Studies of the seafloor conductivity [e.g., Young and Cox, 1981] suggest that a typical conductivity for the first $1 \mathrm{~km}$ of the seabed is 0.1 $\mathrm{S} / \mathrm{m}$. Thus the range of seabed conductivities considered in our computations should cover most practical seabeds.

The field data are presented in six figures, as follows: VED, Figure 2; VMD, Figure 3; HED, $\phi=0^{\circ}$, Figure 4 ; HED, $\phi=90^{\circ}$, Figure 5; HMD, $\phi=0^{\circ}$, Figure 6; and HMD, $\phi=90^{\circ}$, Figure 7. Within each figure, there are four panels on the left providing the field amplitudes in parametric form, and matching panels on the right containing the curves showing the ratios of the field amplitudes produced by the dipole on the seafloor to the amplitudes produced under otherwise identical conditions but with the seabed replaced by seawater $\left(\sigma_{f}=\sigma_{s}\right)$. The ratio curves provide an immediate qualitative indication of the scale of the enhancements, or decreases, of the field amplitudes due to the presence of the seabed, since, as we have noted, the absence of a seabed effect is indicated by a ratio of 1.0 (in the figures, this corresponds to a horizontal line passing through 0 on the vertical axis). In addition, if desired, the curves can be used to give quantitative information about the changes in the fields caused by the seabed.

To provide an example of the use of the data in Figures 2-7, suppose the source of the fields is a VED of moment $10 \mathrm{~A} \mathrm{~m}$ transmitting at $100 \mathrm{~Hz}$ and we wish to know the amplitude of $E_{z}$ at a distance of $r=100 \mathrm{~m}$ on a seabed with an effective conductivity of $0.1 \sigma_{s}$. First, we compute the seawater skin depth $\delta_{s}$ at $100 \mathrm{~Hz}$ (it will be assumed that $\sigma_{s}=4.0 \mathrm{~S} / \mathrm{m}$ ) and obtain $\delta_{s}=25.2 \mathrm{~m}$. Thus $r / \delta_{s}=3.97$. From the panel for $E_{z}$ in Figure 2 we read off $E_{z} \times \delta_{s}^{3} \sigma_{s}=3.0$ $\times 10^{2} \mu \mathrm{V} / \mathrm{m} \times \mathrm{m}^{2} \mathrm{~S}$, or $E_{z}=0.0047 \mu \mathrm{V} / \mathrm{m}$. This electric field amplitude applies to a unit moment dipole; for a dipole of moment $10 \mathrm{~A} \mathrm{~m}$ it is $E_{z}=0.047 \mu \mathrm{V} / \mathrm{m}$. Turning to the ratio curves, we make the perhaps surprising finding that the seabed reduces the amplitude of $E_{z}$ to about 0.35 of its equivalent value in seawater of infinite extent; it is only for distances greater than $10 \delta_{s}$ in this example that the amplitude 

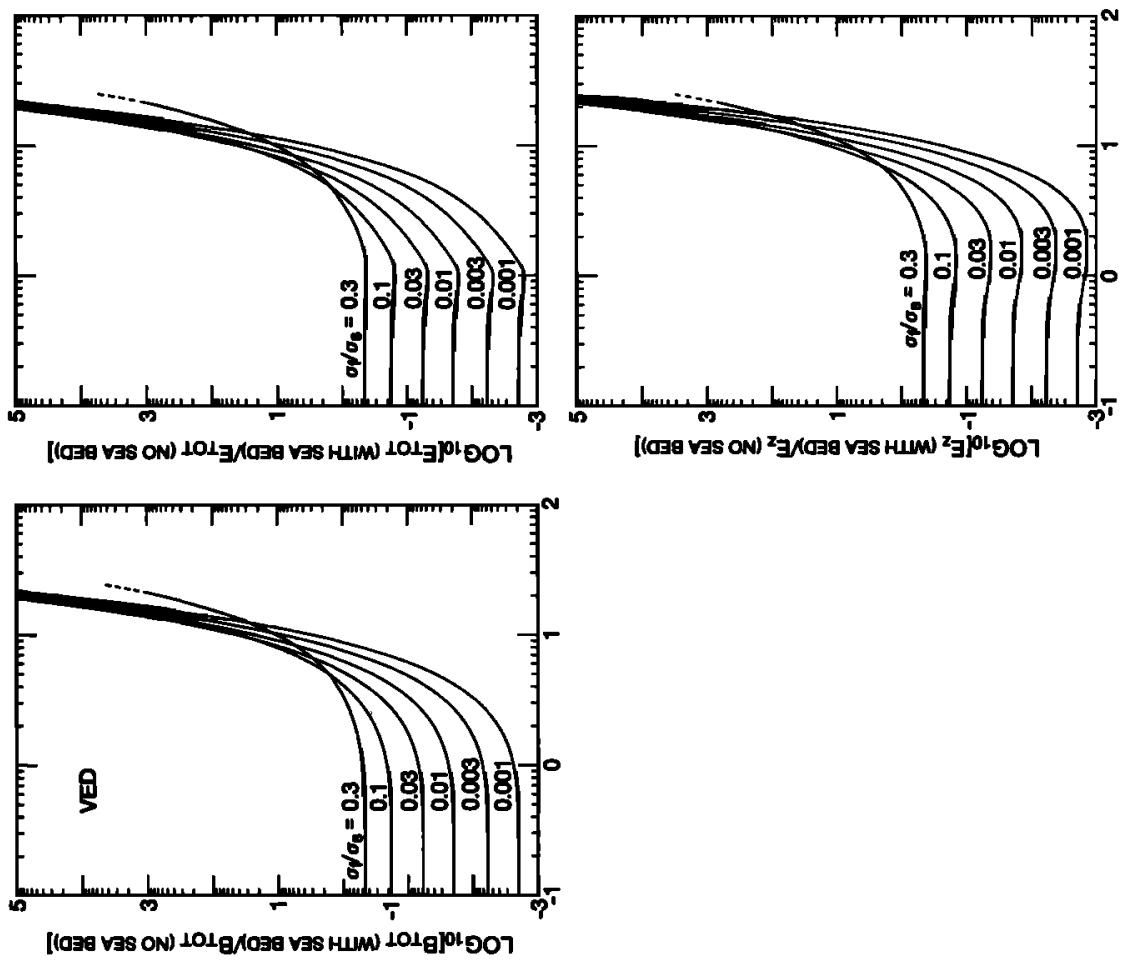

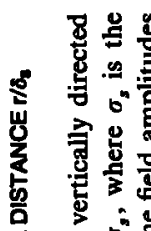

है

80

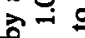

용

$0^{\circ}$

웅

象落

可 른

里

品.

需

要

定

焉

o

is

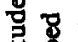

总:
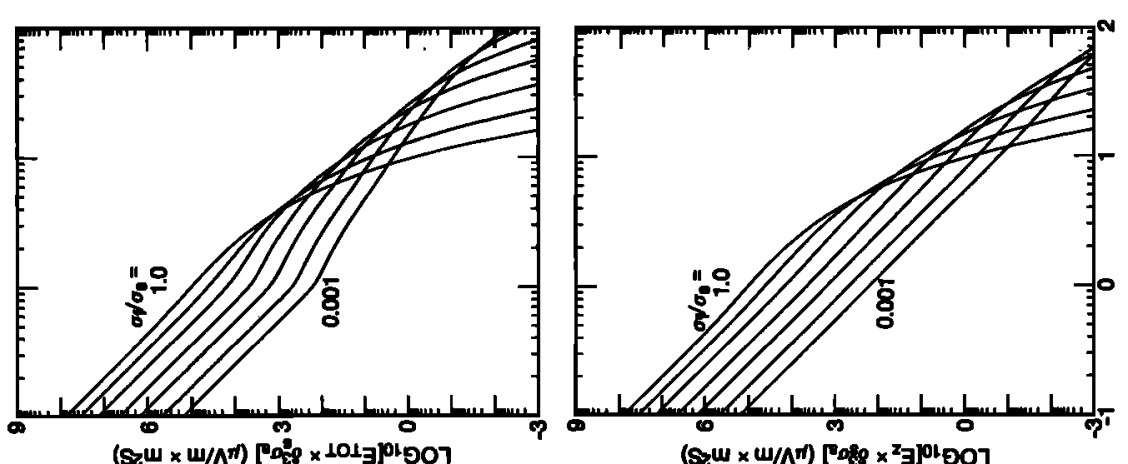

影

象

○

능 뎡

ㅇํㄹ

8

要

离 总高

एँ ब

8 造

爱家远

要安

氙零

응
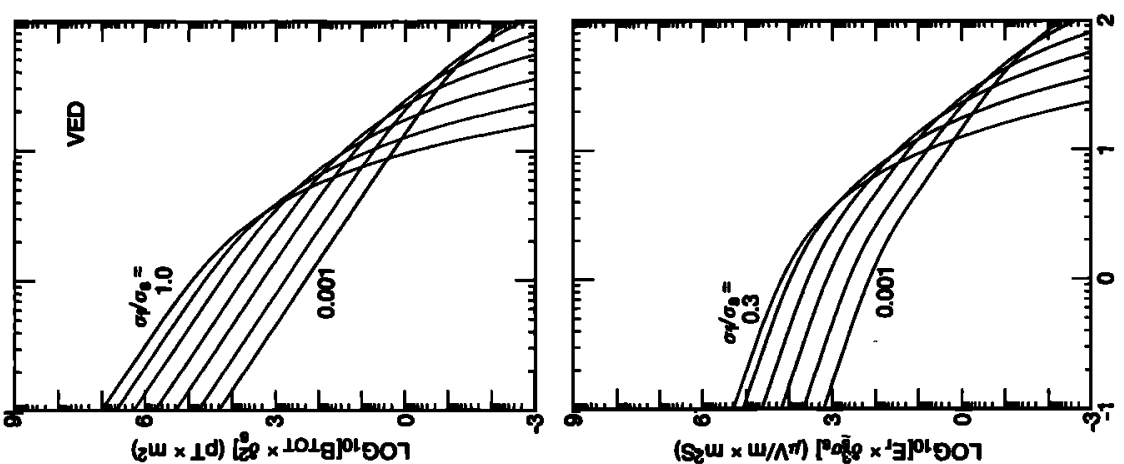

둥

胥蛋

$>\frac{0}{2} \geqslant$

ㄴ.

螘悬总 

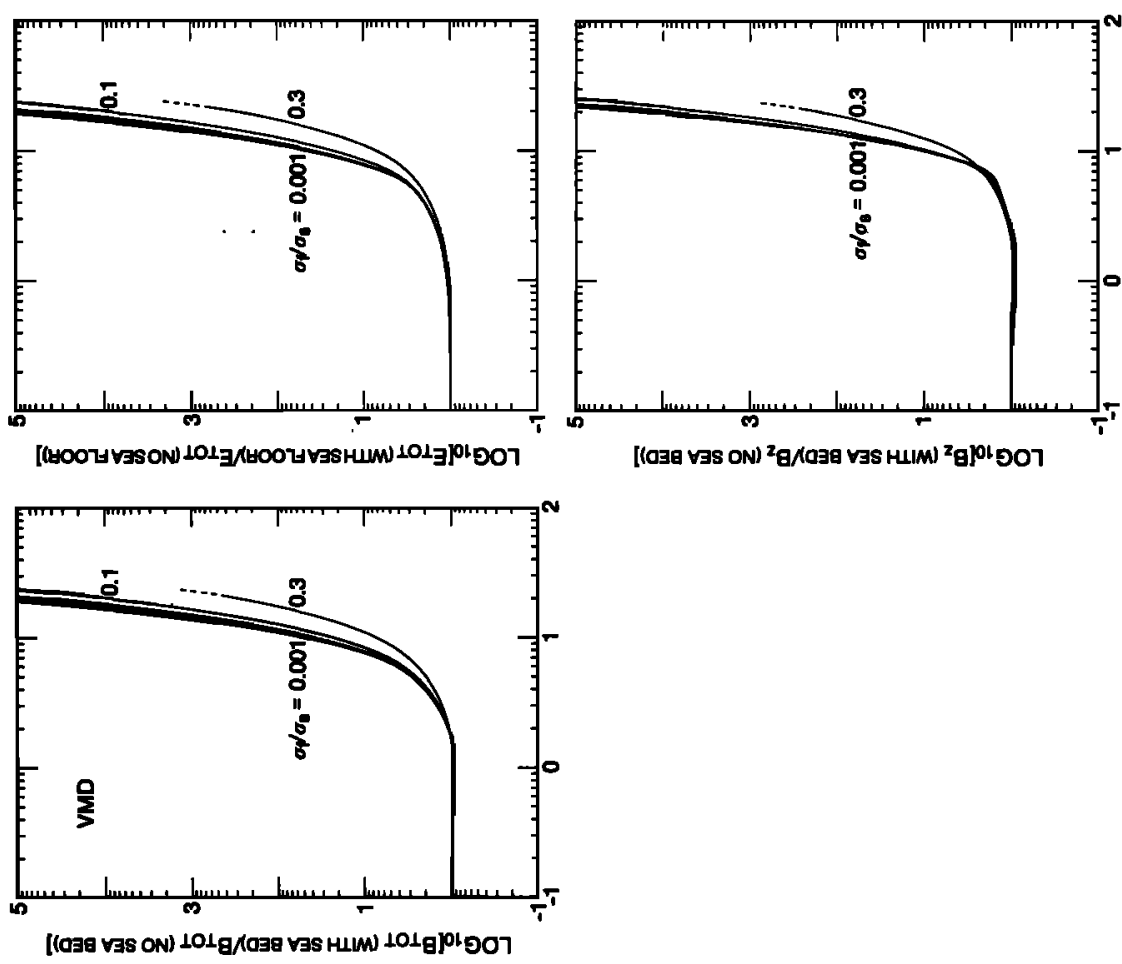

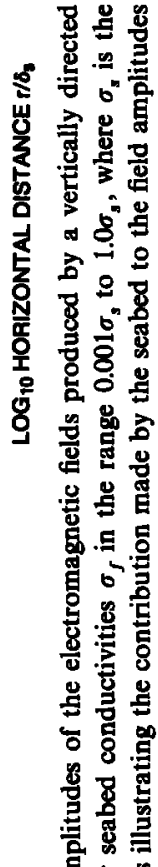
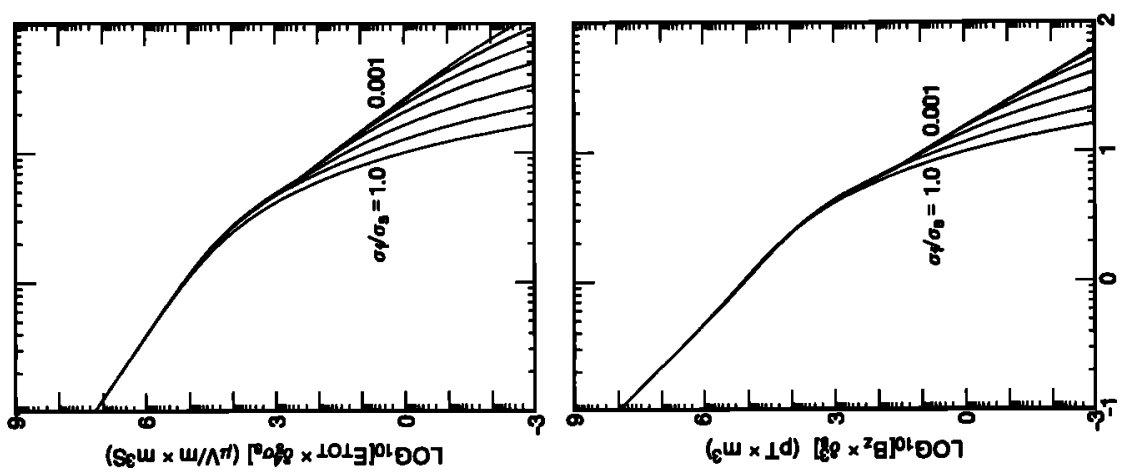

鼠客

象

을 윻

究

过

总

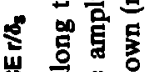

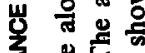

实

兽完是
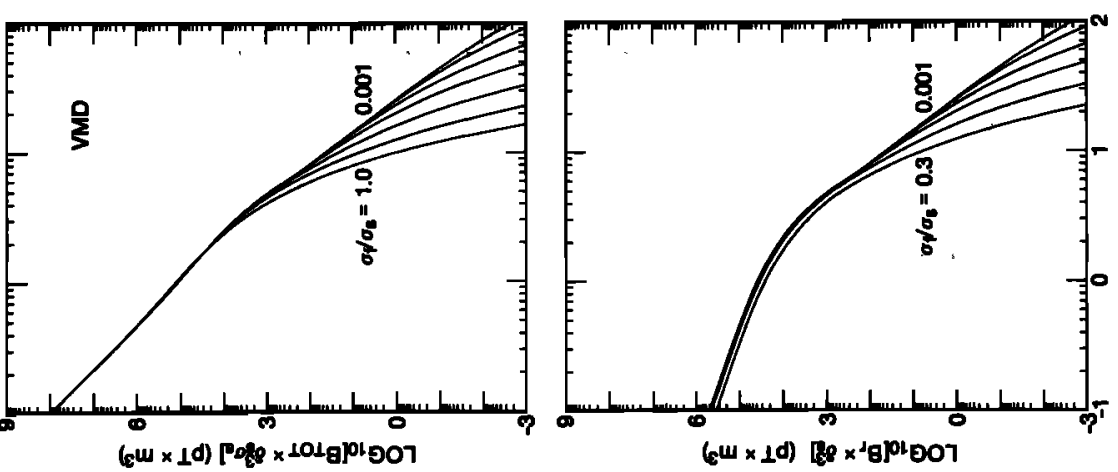

둰

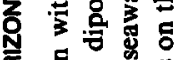

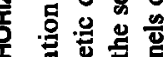

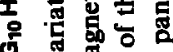

$>$ 초

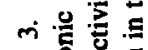

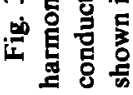



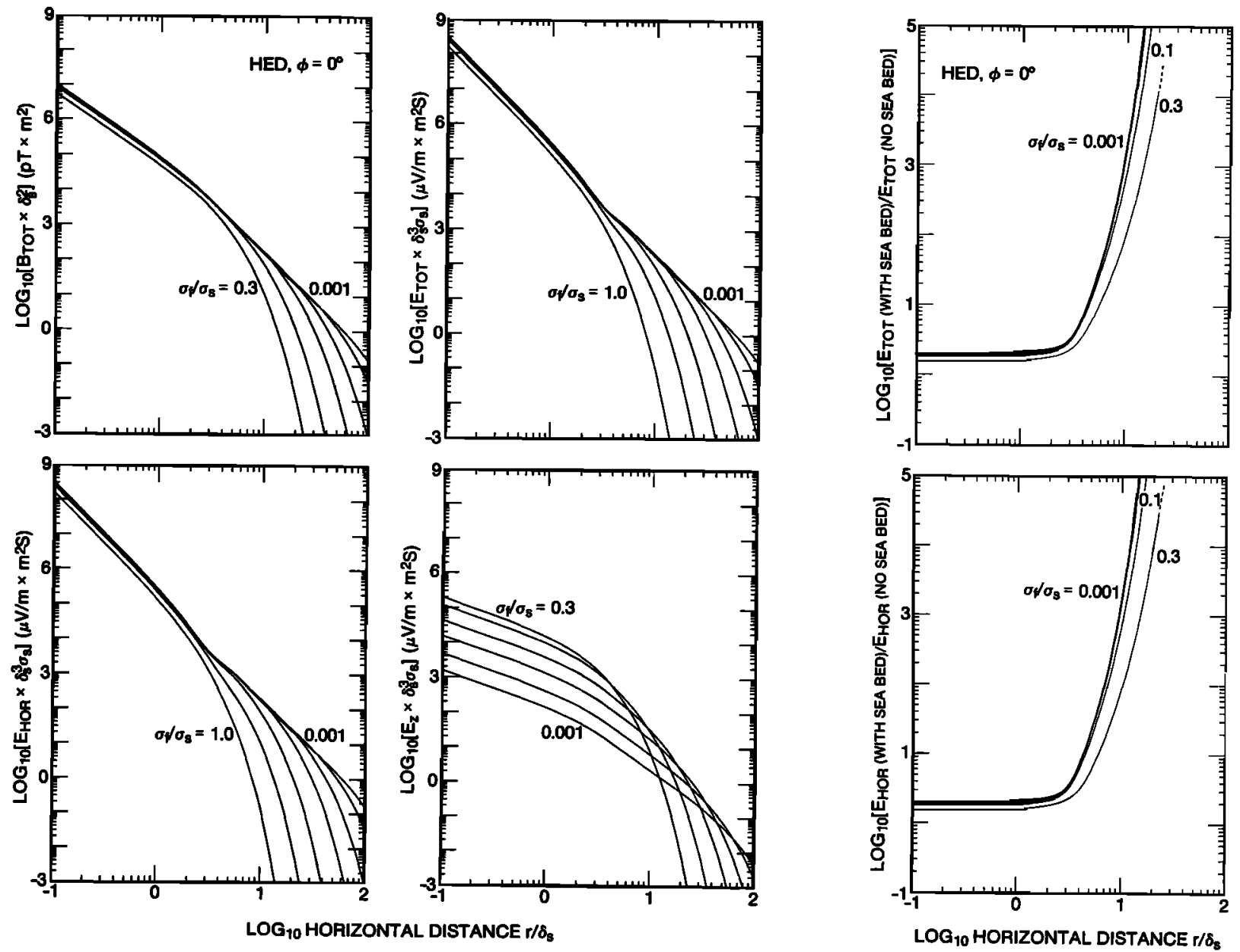

Fig. 4. Variation with distance along the seafloor of the amplitudes of the electromagnetic fields produced at an azimuthal angle $\phi$ of $0^{\circ}$ by a horizontally directed harmonic electric dipole (left). The amplitudes are shown for seabed conductivities $\sigma_{f}$ in the range $0.001 \sigma_{s}$ to $1.0 \sigma_{s}$, where $\sigma_{s}$ is the conductivity of the seawater. Also shown (right) are ratio curves illustrating the contribution made by the seabed to the field amplitudes shown in the panels on the left.

begins to show an increase due to the presence of the seabed, but the increase with increasing distance then becomes very rapid. Finally, if we divide the amplitude of $E_{z}(0.047 \mu \mathrm{V} / \mathrm{m})$ for the unit moment dipole by 0.35 , we obtain a numerical value for the amplitude $(0.134 \mu \mathrm{V} / \mathrm{m})$ that would be produced by the dipole in a sea of infinite extent. The same amplitude can be computed from the appropriate field expression in the set given by Kraichman [1976] for dipoles immersed in an infinite conducting medium, thus providing a check of the results of our numerical computations.

For each dipole category there is at least one and sometimes two (HED, $\phi=0^{\circ}$; and HMD, $\phi=0^{\circ}$ ) matching panels missing on the right-hand sides of the displays. The reason for the gaps is of great interest from the point of view of the effects produced by a seabed. Not only can the seabed change the amplitudes of the field components that would be present in the absence of the bed $\left(\sigma_{f}=\sigma_{s}\right)$, but it can also produce new field components which, in addition, often have amplitudes that are greater than those of the other components at large distances. Because these latter components do not exist in a sea of infinite extent, ratio curves cannot be computed, and gaps are produced in the displays. The missing panels on the right-hand sides of the figures therefore provide a guide to the field quantities that owe their existence to the presence of the seabed. To be specific, the new field quantities are VED, $E_{r} ; \mathrm{VMD}, B_{r}$; 

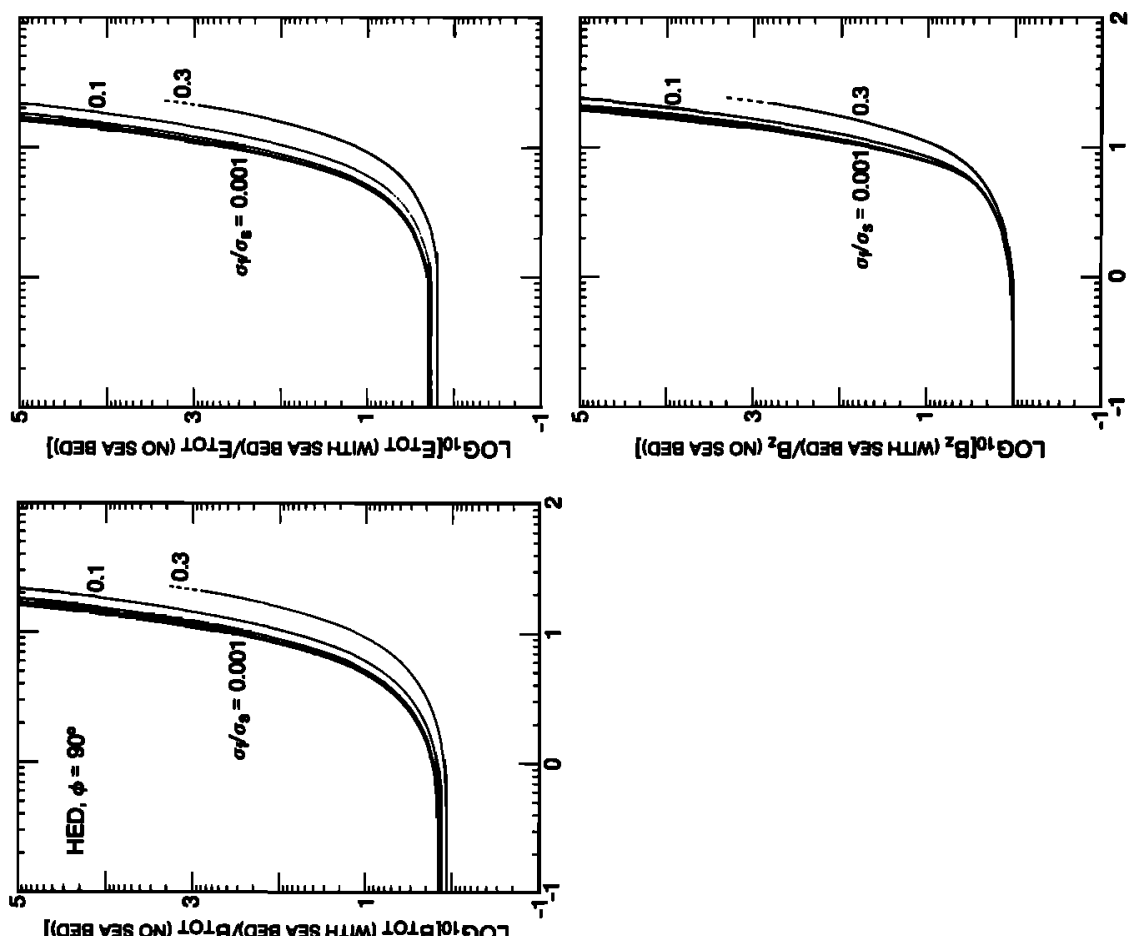

6 을

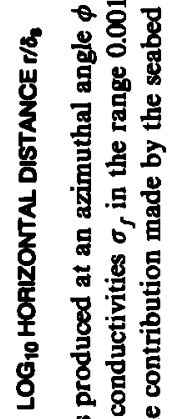

용

过

㝵皆

的当

행

$\frac{8}{5}$ 를

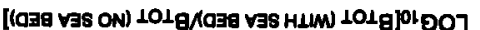

\& 8

影

8릉 늠

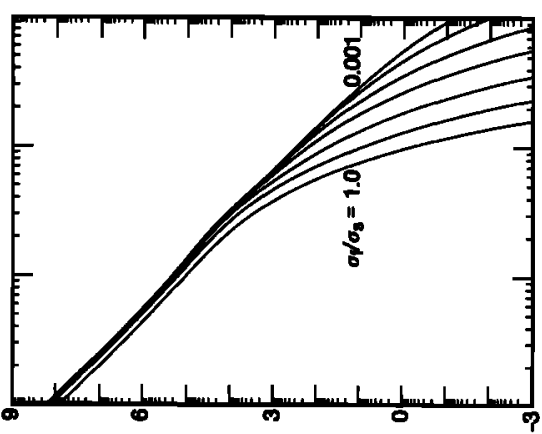

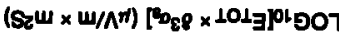
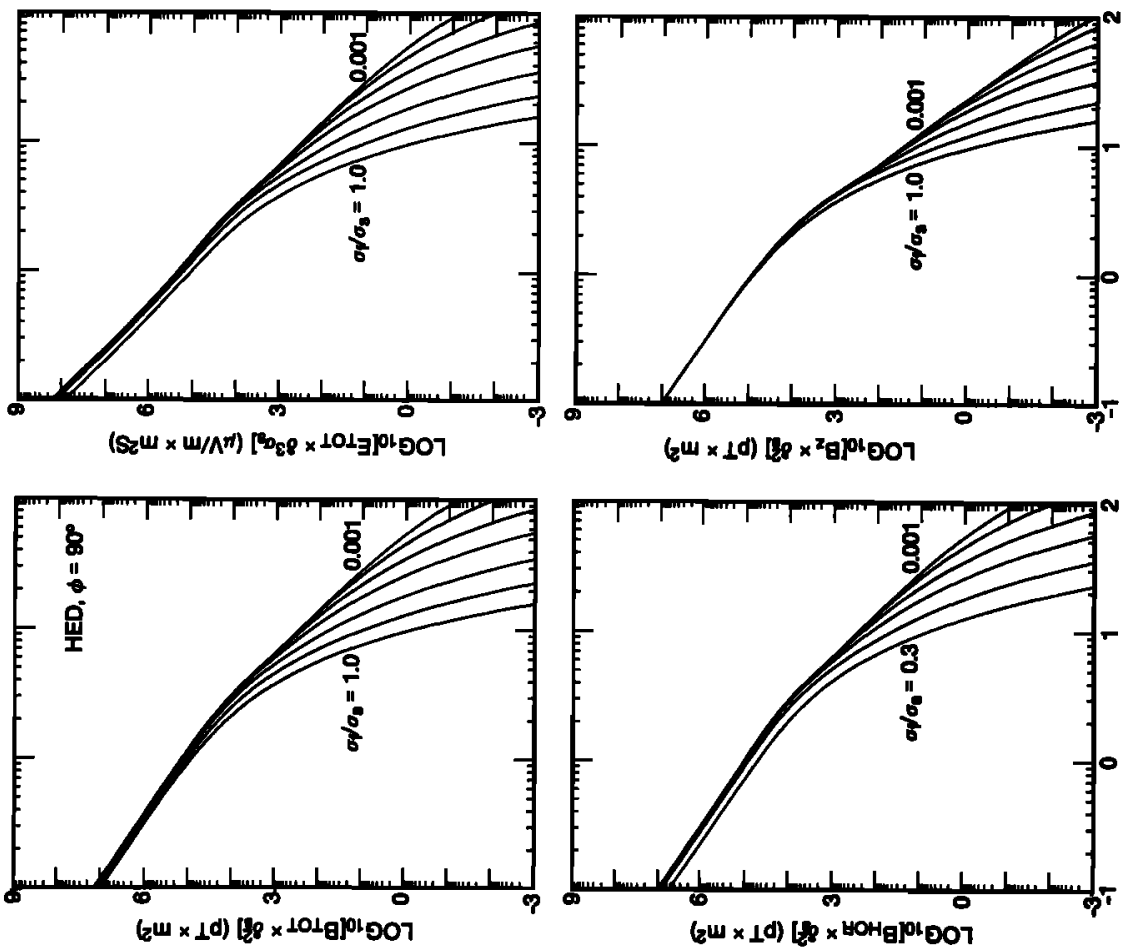

总言

虽是

再

起

능

응

宛它过

ฐ

on

을 흥

8 具

乎起

急 还

동 焉

코옹

西 $0^{n}$

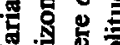

$>$ 突

공

의

\& 9 

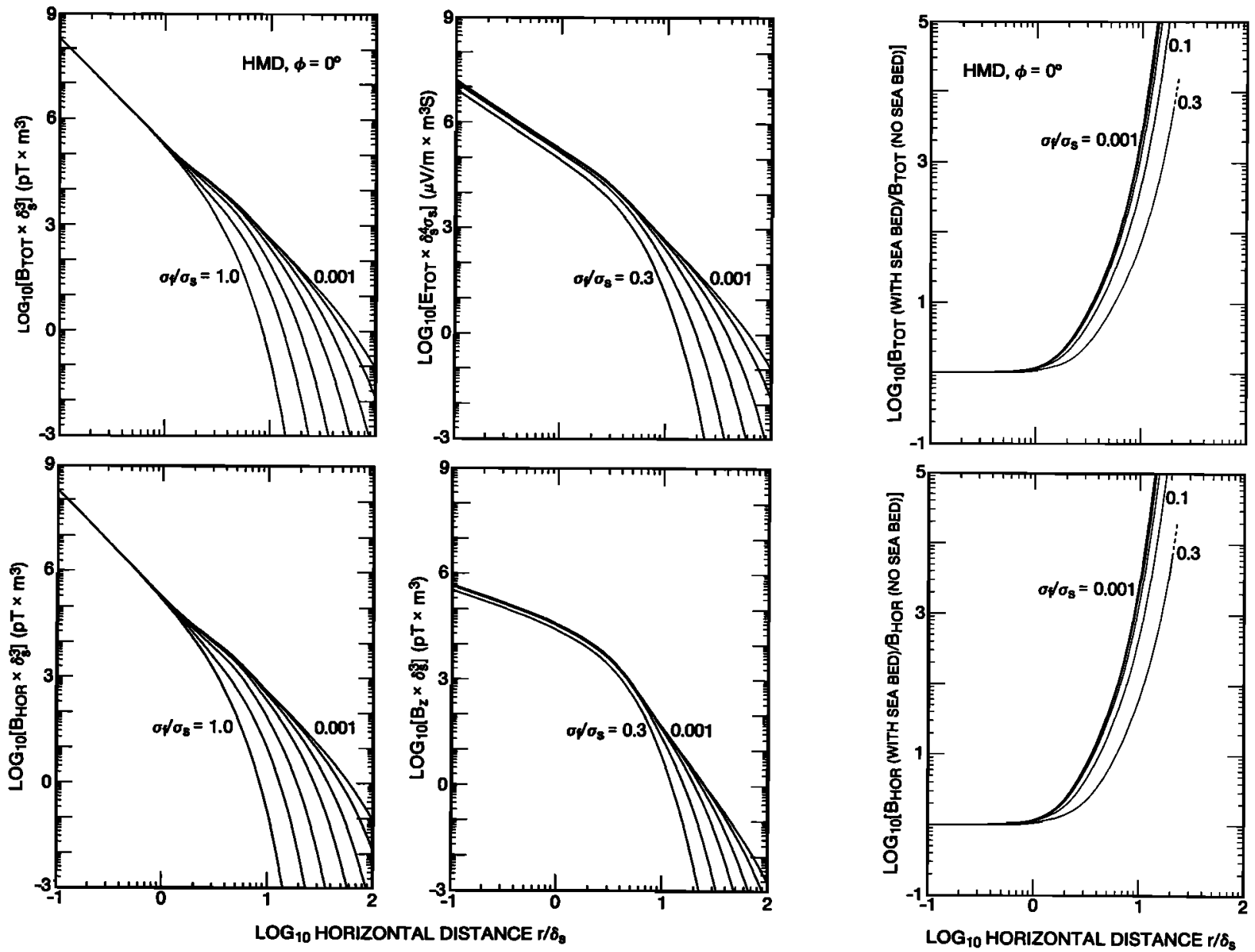

Fig. 6. Variation with distance along the seafloor of the amplitudes of the electromagnetic fields produced at an azimuthal angle $\phi$ of $0^{\circ}$ by a horizontally directed harmonic magnetic dipole (left). The amplitudes are shown for seabed conductivities $\sigma_{f}$ in the range $0.001 \sigma_{s}$ to $1.0 \sigma_{s}$, where $\sigma_{s}$ is the conductivity of the seawater. Also shown (right) are ratio curves illustrating the contribution of the seabed to the field amplitudes shown in the panels on the left.

HED, $\phi=0^{\circ}, E_{z}$ and $B_{\phi}$ (or $B_{\text {TOT }}$ ); HED, $\phi=90^{\circ}, B_{r}$ (or $B_{\mathrm{HOR}}$ ); HMD, $\phi=0^{\circ}, E_{\phi}$ (or $E_{\text {TOT }}$ ) and $B_{z}$; and $\mathrm{HMD}, \phi=90^{\circ}, E_{r}$ (or $E_{\mathrm{HOR}}$ ). In addition to the missing ratio panels for these components, it will be noted that the parametric amplitudes are only plotted for $\sigma_{f} / \sigma_{s} \leq 0.3$, since the components do not exist for $\sigma_{f} / \sigma_{s}=1$.

For comparison with our dipole field data we also computed numerical values for some of the field components produced by an HED located on the seafloor using the approximate expressions given by King $[1985 a, b]$. Specifically, we took the two expressions for the HED electric field component $E_{1 \rho}^{L}$ given by $K$ ing [1985a, equations $(10 b)$ and $(45 a)]$ and calculated the field amplitudes for various seabed conductivities and distances. The most approximate expression, given by King's equation (10b), gives field amplitudes differing from ours and from those given by King's more accurate expression (equation (45a)) by up to a factor of 2 , depending on which part of King's "useful intermediate range" is involved. On the other hand, the more accurate expression gives field values that are in close agreement with ours, particularly within the middle part of the range of applicability of the expression.

In addition to the above comparison, we also computed values of $E_{\phi}$ and $B_{z}$ for the VMD and $B_{z}$ for the HED using exact analytical expressions given by Wait [1952, 1961] and compared the results with those obtained by our numerical integration method. 

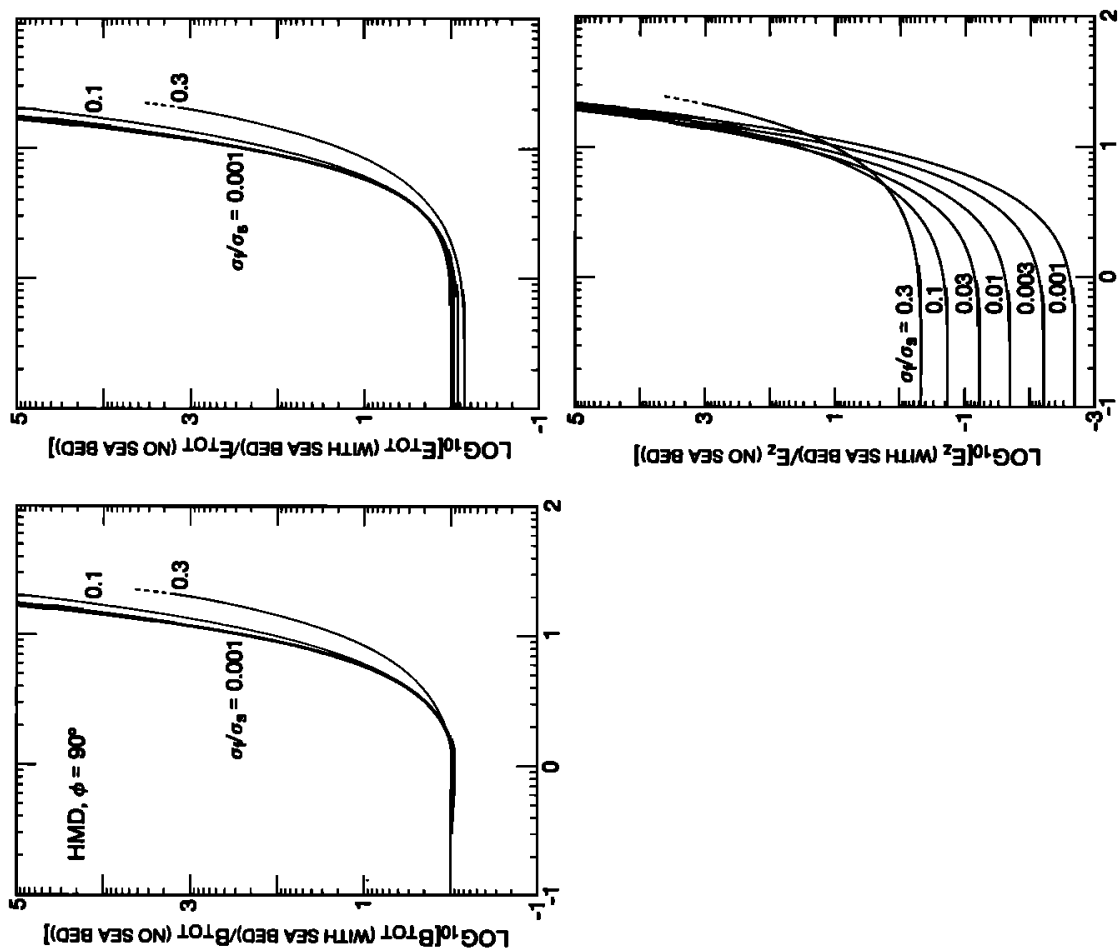

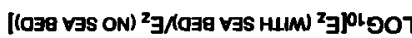
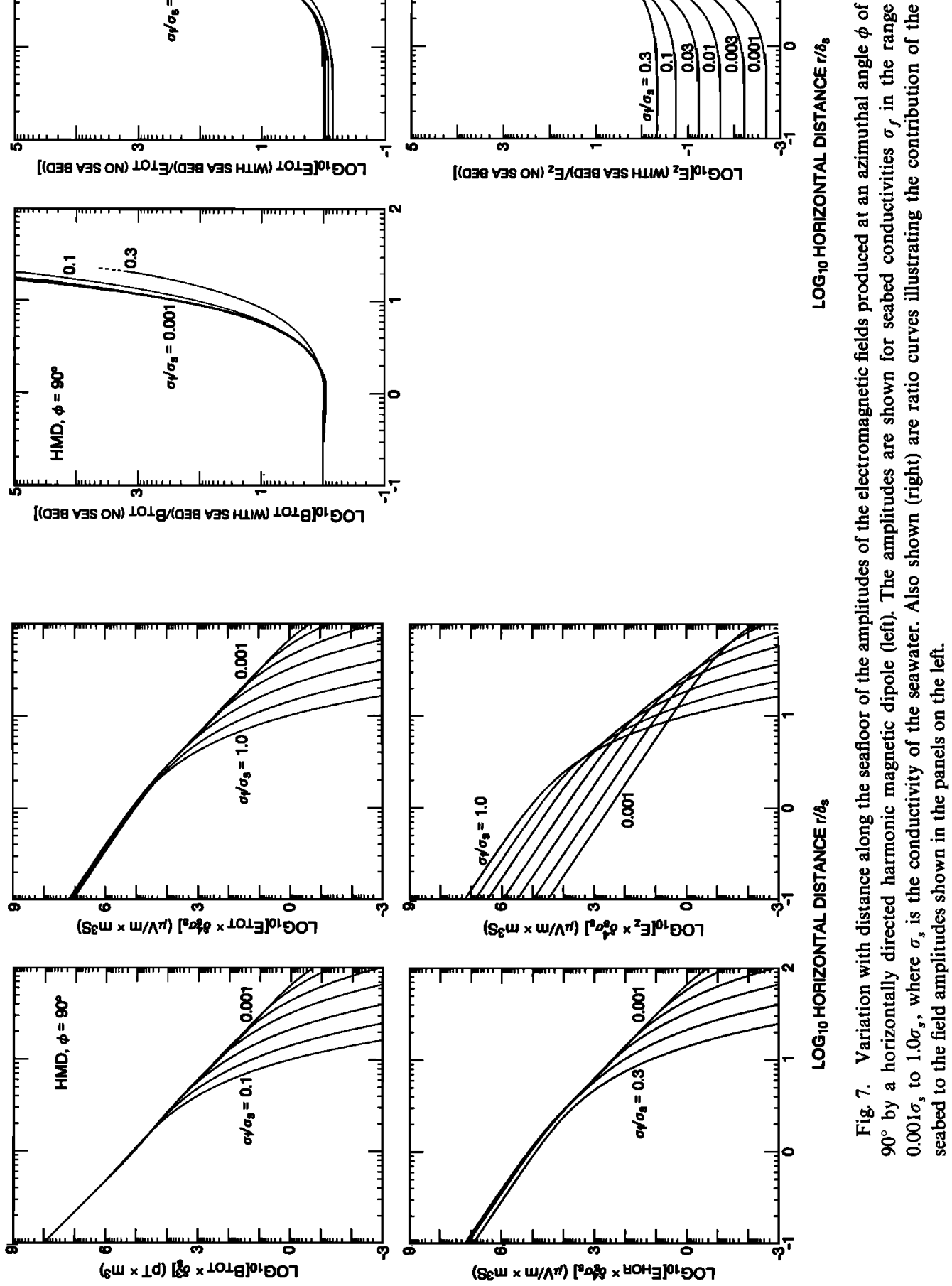
For the distances and seabed conductivities covered by the data in Figures $2-7$ there was excellent agreement between the two sets of field data, usually to many significant figures. However, for combinations of large distances and high seabed conductivities outside those illustrated in the figures, the field quantities became so small that computer rounding errors in the numerical integration method introduced discrepancies. Partly for this reason, and partly to save computation time, the ratio data in the figures were computed using the known analytical expressions for the fields produced in a sea of infinite depth [Kraichman, 1976].

\section{DISCUSSION}

There are a number of interesting general features of the data shown in Figures 2-7. First, as anticipated, there can be substantial increases in the field components produced along the seafloor in comparison to those that would be produced under otherwise identical conditions in a sea of infinite extent. These increases only occur for horizontal distances greater than about $3 \delta_{s}$, but they then grow rapidly with distance. Increases of 4 orders of magnitude or more at distances of $20 \delta_{s}$ are typical. Second, for field amplitudes that are approaching the limit of detection of present measurement systems, the presence of the seabed can increase the range of detection of the fields by roughly 2-10 times, depending on the effective conductivity of the seabed. Third, as we have already noted, additional field components are produced when a seabed is present (in comparison to a sea of infinite depth), and some of these additional components predominate at larger distances. Finally, fourth, the ratio curves tend to be very similar for the range of seabed conductivities covered by our computations $\left(\sigma_{f} / \sigma_{s}=0.001-0.3 \mathrm{~S} / \mathrm{m}\right)$. Nevertheless, we know that the curves transform into a horizontal line passing through 0 on the vertical axis as $\sigma_{f} / \sigma_{s} \rightarrow 1$, and there is indeed some evidence for this transformation when the ratio curves for $\sigma_{f} / \sigma_{s}=0.1$ and 0.3 are compared with the others. Our interpretation of this result is that the field amplitudes produced at distances greater than about $5 \delta_{s}$ are particularly sensitive to seabed conductivities in the range $0.1 \sigma_{s}$ to $1.0 \sigma_{s}$. Conversely, except for the VED fields and $E_{z}$ for the HMD, $\phi=90^{\circ}$, the field amplitudes tend not to be very sensitive to seabed conductivities less than about $0.1 \sigma_{s}$.

Comparing the fields generated along the seafloor, we see that there are some major differences between dipole types. In particular, for low seabed conductivities, the HED and HMD produce much larger fields at large distances than do the VED and VMD. The difference is substantial, amounting to 2 orders of magnitude at a distance of $100 \delta_{s}$ for $\sigma_{f} / \sigma_{s}=0.001$. This result is in agreement with the more restricted observation by Frieman and Kroll [1973] that an HED was far superior to a VED for producing ULF fields along the seafloor. We might also comment that the VED also appears inferior to other dipole types for producing fields at short to moderate distances $\left(r<10 \delta_{s}\right)$, particularly when the seabed has a low effective conductivity.

In conclusion, this study makes evident the important role that the seafloor could play in undersea communication by means of freely propagating ULF/ELF electromagnetic waves from harmonic dipole sources located on or near the seafloor. The combination of possible large seabed enhancements of the fields, comparatively low noise levels from atmospheric sources, and a fixed surface on which repeaters can be located could well make feasible the utilization of the seabed as a communication medium.

Acknowledgments. We thank D. M. Bubenik for the use of his computer code and P. R. Bannister for helpful discussions. This work was supported by the Office of Naval Research under contract N00014-83-K-0390.

\section{REFERENCES}

Bannister, P. R., Determination of the electrical conductivity of the sea bed in shallow waters, Geophysics, 33, 995-1003, 1968.

Bostick, F. X., C. S. Cox, and E. C. Field, Jr., Land-to-seafloor electromagnetic transmissions in the 0.1 to $15 \mathrm{~Hz}$ band, Radio Sci., 13, 701-708, 1978.

Bubenik, D. M., A practical method for the numerical evaluation of Sommerfeld integrals, IEEE Trans. Antennas Propag., AP-25, 904-906, 1977.

Bubenik, D. M., and A. C. Fraser-Smith, ULF/ELF electromagnetic fields generated in a sea of finite depth by a submerged vertically-directed harmonic magnetic dipole, Radio Sci., 13, 1011-1020, 1978.

Burrows, C. R., Radio communication within the Earth's crust, IEEE Trans. Antennas Propag., AP-25, 311-317, 1963.

Coggon, J. H., and H. F. Morrison, Electromagnetic investigation of the sea floor, Geophysics, 35, 476-489, 1970.

Fraser-Smith, A. C., and D. M. Bubenik, ULF/ELF electromagnetic fields generated above a sea of finite depth by a submerged vertically-directed harmonic magnetic dipole, Radio Sci., 14, 59-74, 1979.

Fraser-Smith, A. C., and D. M. Bubenik, Compendium of the ULF/ELF electromagnetic fields generated above a sea of finite depth by submerged harmonic dipoles, Tech. Rep. E715-1, 114 pp., Radiosci. Lab., Stanford Univ., Stanford, Calif., 1980. 
Fraser-Smith, A. C., D. M. Bubenik, and O. G. Villard, Jr., Largeamplitude changes induced by a seabed in the sub-LF electromagnetic fields produced in, on, and above the sea by harmonic dipole sources, Radio Sci., 22, 567-577, 1987.

Frieman, E. A., and N. M. Kroll, Lithospheric propagation for undersea communication, Tech. Rep. JSR-73-5, 23 pp., Stanford Res. Inst., Menlo Park, Calif., 1973.

Gabillard, R., P. Degauque, and J. R. Wait, Subsurface electromagnetic telecommunication-A review, IEEE Trans. Commun. Technol., COM-19, 1217-1228, 1971.

Hansen, R. C., Radiation and reception with buried and submerged antennas, IEEE Trans. Antennas Propag., AP-11, 207 $216,1963$.

Heacock, J. G. (Ed.), The Structure and Physical Properties of the Earth's Crust, Geophys. Monogr. Ser., vol. 14, 348 pp., AGU, Washington, D. C., 1971.

Inan, A. S., Propagation of electromagnetic fields along the sea/sea-bed interface, Tech. Rep. E721-2, 70 pp., Space, Telecommun. and Radiosci. Lab., Stanford Univ., Stanford, Calif., 1984.

Inan, A. S., A. C. Fraser-Smith, and O. G. Villard, Jr., ULF/ELF electromagnetic fields generated along the seafloor interface by a straight current source of infinite length, Radio Sci., 21, 409$420,1986$.

King, R. W. P., Electromagnetic surface waves: New formulas and applications, IEEE Trans. Antennas Propag., AP-33, 1204-1212, $1985 a$.

King, R. W. P., Electromagnetic surface waves: New formulas and their application to determine the electrical properties of the sea bottom, J. Appl. Phys., 58, 3612-3624, $1985 b$.

King, R. W. P., Properties of the lateral electromagnetic field of a vertical dipole and their application, IEEE Trans. Geosci. Remote Sens., GE-24, 813-825, 1986.

King, R. W. P., and M. F. Brown, Lateral electromagnetic waves along plane boundaries: A summarizing approach, Proc, IEEE, 72, 595-611, 1984.

King, R. W. P., M. Owens, and T. T. Wu, Properties of lateral electromagnetic fields and their application, Radio Sci., 21, 13-23, 1986.

Kraichman, M. B., Handbook of Electromagnetic Propagation in Conducting Media, U.S. Government Printing Office, Washington, D. C., 1976.
Moore, R. K., Radio communication in the sea, IEEE Spectrum, 4, 42-51, 1967.

Moore, R. K., and W. E. Blair, Dipole radiation in a conducting half-space, J. Res. Natl. Bur. Stand., Sect. D, 65, 547-563, 1961.

Mott, H., and A. W. Biggs, Very-low-frequency propagation below the bottom of the sea, IEEE Trans. Antennas Propag., AP-11, 323-329, 1963.

Ramaswamy, V., H. W. Dosso, and J. T. Weaver, Horizontal magnetic dipole embedded in a two-layer conducting medium, $\mathrm{Can}$. J. Phys., 50, 607-616, 1972.

Wait, J. R., Current-carrying wire loops in a simple inhomogeneous region, J. Appl. Phys., 23, 497-498, 1952.

Wait, J. R., On anomalous propagation of radio waves in earth strata, Geophysics, 19, 342-343, 1954.

Wait, J. R., The electromagnetic fields of a horizontal dipole in the presence of a conducting half-space, Can. J. Phys., 39, 1017$1028,1961$.

Wait, J. R., and K. P. Spies, Electromagnetic propagation in an idealized earth crust waveguide, I, Pure Appl. Geophys., 101, 174-187, 1972a.

Wait, J. R., and K. P. Spies, Electromagnetic propagation in an idealized earth crust waveguide, II, Pure Appl. Geophys., 101, 188-193, 1972b.

Wait, J. R., and K. P. Spies, Dipole excitation of ultra-lowfrequency electromagnetic waves in the earth crust waveguide, J. Geophys. Res., 77, 7118-7120, 1972c.

Weaver, J. T., The quasi-static field of an electric dipole embedded in a two-layer conducting half-space, Can. J. Phys., 45, 1981$2002,1967$.

Wheeler, H. A., Radio-wave propagation in the earth's crust, $J$. Res. Natl. Bur. Stand., Sect. D, 65, 189-191, 1961.

Young, P. D., and C. S. Cox, Electromagnetic active source sounding near the East Pacific Rise, Geophys. Res. Lett., 8, 1043-1046, 1981.

A. C. Fraser-Smith and O. G. Villard, Jr., STAR Laboratory, Stanford University, Stanford, CA 94305.

A. S. Inan, Department of Electrical and Electronics Engineering, Bilkent University, Ankara, Turkey.

R. G. Joiner, Office of Naval Research, Arlington, VA 22217. 